\title{
An Image Watermarking Scheme Using Arnold Transform and Fuzzy Smooth Support Vector Machine
}

\author{
Lin Sun, ${ }^{1,2,3}$ Jiucheng Xu, ${ }^{1,2,3}$ Xingxing Zhang, ${ }^{1}$ and Yun Tian ${ }^{4}$ \\ ${ }^{1}$ College of Computer and Information Engineering, Henan Normal University, Henan 453007, China \\ ${ }^{2}$ Engineering Technology Research Center for Computing Intelligence and Data Mining, Henan 453007, China \\ ${ }^{3}$ Engineering Laboratory of Intellectual Business and Internet of Things Technologies, Henan 453007, China \\ ${ }^{4}$ College of Information Science and Technology, Beijing Normal University, Beijing 100875, China
}

Correspondence should be addressed to Lin Sun; linsunok@gmail.com

Received 10 October 2014; Revised 2 January 2015; Accepted 5 January 2015

Academic Editor: Daniel Zaldivar

Copyright (C) 2015 Lin Sun et al. This is an open access article distributed under the Creative Commons Attribution License, which permits unrestricted use, distribution, and reproduction in any medium, provided the original work is properly cited.

\begin{abstract}
With the development of information security, the traditional encryption algorithm for image has been far from ensuring the security of image in the transmission. This paper presents a new image watermarking scheme based on Arnold Transform (AT) and Fuzzy Smooth Support Vector Machine (FSSVM). First of all, improved AT (IAT) is obtained by adding variables and expanding transformation space, and FSSVM is proposed by introducing fuzzy membership degree. The embedding positions of watermark are obtained from IAT, and the pixel values are embedded in carrier image by quantization embedding rules. Then, the watermark can be embedded in carrier image. In order to realize blind extraction of watermark, FSSVM model is used to find the embedding positions of watermark, and the pixel values are extracted by using quantization extraction rules. Through using improved Arnold inverse transformation for embedding positions, the watermark coordinates can be calculated, and the extraction of watermark is carried out. Compared with other watermarking techniques, the presented scheme can promote the security by adding more secret keys, and the imperceptibility of watermark is improved by introducing quantization rules. The experimental results show that the proposed method outperforms many existing methods against various types of attacks.
\end{abstract}

\section{Introduction}

Application and popularization of multimedia technologies and computer networks have made duplication and distribution much easier for multimedia contents $[1,2]$. The digital media (such as video, image, audio, and text) can be modified easily by attackers who can claim their ownership [3]. Then copyright protection of intellectual properties has become an important and challenging task. One way for copyright protection is digital watermarking, which means embedding certain specific information about the copyright holder into the protected media [4]. In the last decade, digital watermarking methods for images from different purposes are usually categorized into two types: robust watermarking and fragile watermarking. And the watermarking techniques can also be classified into two groups based on the domain in which the watermark is inserted: spatial domain techniques and frequency domain techniques [2,5-7]. The methods of spatial domain have advantages of easy implementation and low cost of operation, but they are generally not robust to geometrical attacks and image processing. Nowadays, there are many transform domain watermarking techniques $[8,9]$, such as discrete cosine transform, singular value decomposition, discrete Fourier transform, and discrete wavelet transform. In practice, the performances of watermarking methods are further improved by combining two or more transforms [5]. The image encryption using gyrator transform based on two-step phase-shifting interferometry and AT has been proposed in [10]. Liu et al. [11] proposed an image encryption algorithm by using AT and discrete cosine transform. Abuturab [12] proposed the color information encryption in the gyrator transform domain not only based on discrete cosine transform and radial Hilbert phase mask but also based on AT. Sui and Gao [10] presented a color image 
encryption scheme by using gyrator transform and AT. Chen et al. [13] designed a new image encryption algorithm based on singular value decomposition and AT. Chen et al. [14] offered a watermarking scheme based on Arnold cat map. AT has periodicity and the transform is simple, but the periodicity depends on image size. Moreover, it is the main concern of the watermarking schemes that the embedded watermark should not degrade the quality of carrier image and the inserted watermark must be as much invisible as possible. The distortion of the watermarked image should be negligible without degrading its robustness under attacking conditions. In order to generate the watermarked images of high quality and extract high fidelity watermarks in attacking environments, the overall watermarking procedure depends on a set of configuration parameters which need to be optimized. Most existing image watermarking algorithms take scaling factor as a single value which needs the proper fine tune. However, when each singular value has a different tolerance limit, a single scaling factor is not suitable in case of singular value decomposition. It is known that imperceptibility and robustness are two important issues of image watermarking schemes. Then the tradeoff between imperceptibility and robustness in watermarking problems is viewed as an optimization problem.

Recently, attacks against image watermarking systems have become more sophisticated $[15,16]$. A simple solution consists in embedding the watermark in a geometrical invariant subspace, and another strategy for coping with geometric distortions is to insert an additional watermark or template into the carrier image $[17,18]$. However, the implementation difficulties hinder the research of image watermarking schemes based on this principle above. Nasir et al. [19] proposed feature-based image watermarking schemes. Wang et al. [20] proposed a feature-based digital watermarking method for halftone images. However, some drawbacks indwelled in current feature-based schemes restrict the performance of watermarking systems. To address the issues, support vector machine (SVM) is introduced to the image watermarking domain. Fu et al. [21] embedded template and watermark into original images; then the output of SVM models was constructed and the watermark was extracted. Tsai and Sun [22] employed the classification technique based on SVMs to extract a watermark in spatial domain. Li et al. [23] used support vector regression for watermark embedding and extracting in spatial domain. Peng et al. [24] proposed an image watermarking method in multiwavelet domain based on SVMs. Tsai et al. [25] presented a robust lossless watermarking technique based on $\alpha$-trimmed mean algorithm and SVM. Li et al. [26] introduced a semifragile watermarking scheme based on SVM. Yang et al. [17] proposed a new geometrically invariant image watermarking algorithm based on fuzzy SVM correction. However, by virtue of the good learning ability of SVM and AT, most of the existing watermarking schemes mentioned above still have some shortcomings as follows. (1) The standard SVM is always used as a learning scheme, while the speed and precision of training samples are not very ideal. Then, the distortion of final extracted watermark is more serious. (2) As many AT schemes only have single secret key, this characteristic has the limitations of being susceptible to one or several joint attacks and breaks, and then the difficulty of watermark extraction will be increased. (3) Because the extracted image feature is not very stable, hyperplane and generalization capability in SVM of the learned models are affected. Moreover, all samples in training data set are treated uniformly in the same class during the learning process of SVM, but this is not always true. (4) Due to the poor feature vectors, these methods are not very robust against some attacks. In watermark detection procedure, the original carrier image is usually needed, so it is unfavorable to practical application. Furthermore, some of them lack blind detection features; then they cannot balance imperceptibility and robustness effectively.

In this paper, embedding and extraction models of watermark based on AT and FSSVM are proposed with corresponding algorithms. The watermark image is first processed by IAT, and the pixel values are embedded by using quantization embedding rules. The watermarked carrier image can be obtained. Then the embedding method is presented by increasing secret keys to enhance the security degree. The quantization embedding rules can improve imperceptibility of watermark well. FSSVM model is constructed by training some embedding positions to find out positions embedded with watermark in the extraction process. The pixel values of watermark are extracted by introducing quantization extraction rules. FSSVM model not only enhances the training speed and precision of image characteristic values, but also realizes the blind extraction of watermark with the help of quantization extraction rules. The watermark coordinates are calculated by improving Arnold inverse transformation for embedding positions. The original watermark image is extracted, and the processing does not need original carrier image. Moreover, the combination of spatial domain and SVM can balance imperceptibility and robustness of watermark well.

The rest of this paper is organized as follows. In Section 2, AT and SVM techniques are briefly described. In Section 3, the proposed techniques are illustrated. In Section 4, the experimental results are presented and the comparative analysis of our scheme with other methods is given. Finally, the conclusion is drawn in Section 5.

\section{Preliminaries}

2.1. Arnold Transform (AT). In practical applications, AT not only scrambles the pixel position by encoding the iterative number of the process, but also reduces the key spaces of storage and transmission. Although there are many ways for scrambling, here we will discuss only the AT in $[10,14]$, which is an iterative process of moving the pixel position. Suppose that the original image is a $N \times N$ array and the coordinate of the pixel is $F=\{(x, y) \mid x, y=0,1,2, \ldots, N-1\}$. The generalized two-dimension AT is denoted by

$$
\left[\begin{array}{l}
x_{n} \\
y_{n}
\end{array}\right]=\left[\begin{array}{cc}
1 & a \\
b & a b+1
\end{array}\right]\left[\begin{array}{l}
x_{n-1} \\
y_{n-1}
\end{array}\right] \bmod N
$$

where $x_{n}$ and $y_{n}$ are the transformed coordinates corresponding to $x_{n-1}$ and $y_{n-1}$ after $n$ iterations, respectively, $a$ and $b$ are 
positive integers, and $N$ is the height or width of the square image processed.

Since the transformation is an iterative process, if the location $(x, y)$ is transformed several times, it returns to its original position after $T$ iterations. $T$ is called the period of the transformation and depends on parameters $a, b$, and $N$. These parameters $(a, b$, and $N)$ can be used as secret keys in this paper. The pixels will continue to move until they return back to their original positions. Here, the moving time is $T$, and the size of pixel space is $n=0,1,2, \ldots, N-1$. Pixels move with periodicity. $T, a, b$, and $N$ (the size of original watermark) are correlated. Whenever the values change, it generates a completely different Arnold cat map. After being multiplied a few times, the correlation among the pixels will be completely chaotic. To get back to the original image, periodicity is required. Suppose that the scrambling has performed $n$ iterations; then one can get back to the original image by performing $(T-n)$ iterations.

2.2. Support Vector Machine (SVM). SVM has been successfully applied to classification and function estimation problems introduced by Vapnik within the context of statistical learning theory and structural risk minimization [21, 22], and it can be used as regression prediction. Suppose that $\left(x_{1}, y_{1}\right),\left(x_{2}, y_{2}\right), \ldots,\left(x_{l}, y_{l}\right)$ are training samples, and samples have nonlinear relationship in many cases. The regression function can be denoted by $f(x)=w \phi(x)+b$, where $x \in$ $R^{n}$ is an input vector, $w \in R^{n}$ is a weight vector, $\phi$ is a nonlinearity mapping function, and $b \in R$ is an offset.

In order to obtain the last two parameters $w$ and $b$, the structural risk minimization rule is used and the original problem is transformed as follows:

$$
\begin{array}{cc}
\min & \left(\frac{1}{2}\|w\|^{2}+C \sum_{i=1}^{l}\left(\xi_{i}+\xi_{i}^{*}\right)\right), \\
\text { s.t. } & y_{i}-w_{i}-b \leq \varepsilon+\xi_{i} \\
& w_{i}+b-y_{i} \leq \varepsilon+\xi_{i}^{*}, \\
\xi_{i}, \xi_{i}^{*} \geq 0
\end{array}
$$

where $\|w\|^{2}$ describes the complexity of the function $f, C>$ 0 is a constant to determine the complex rate of model and moderation of experimental risk, $\xi_{i}$ and $\xi_{i}^{*}$ are slack variables, $y_{i}$ is a sample, $w_{i}$ is a weight vector, $b \in R$ is an offset, and $\varepsilon$ is an insensitive loss function.

To solve the above convex optimization problem, the core idea is to transform the optimization question into dual form by using Lagrange multiplier method as follows:

$$
\begin{array}{r}
\min \quad\left(-\frac{1}{2} \sum_{i, j=1}^{l}\left(\alpha_{i}-\alpha_{i}^{*}\right)\left(\alpha_{j}-\alpha_{j}^{*}\right)\left(x_{i} \cdot x_{j}\right)\right. \\
\left.\quad-\varepsilon \sum_{i=1}^{l}\left(\alpha_{i}-\alpha_{j}^{*}\right)+\sum_{i=1}^{l} y_{i}\left(\alpha_{i}-\alpha_{i}^{*}\right)\right), \\
\text { s.t. } \quad \sum_{i=1}^{l}\left(\alpha_{i}-\alpha_{i}^{*}\right)=0, \quad \alpha_{i}, \alpha_{i}^{*} \in[0, C],
\end{array}
$$

where $\alpha_{i}, \alpha_{i}^{*}, \alpha_{j}$, and $\alpha_{j}^{*}$ are the weight coefficients and $\bullet$ denotes the inner product operation. In the above formulas, kernel function is introduced to complete the inner product computation for inputting data of high-dimensional feature space while the $\phi$ is not known. The kernel function must satisfy Mercer theorem.

\section{Proposed Techniques}

3.1. Fuzzy Smooth Support Vector Machine (FSSVM). In order to improve the efficiency and precision of prediction, SVM is introduced, and through combining with fuzzy mathematics and transforming the problem into unconstrained optimization problem, one can optimize the object function and transform the risk function into fuzzy dual extreme problems. Then, it can effectively reduce the errors between the predicted pixel values and the actual pixel values in the carrier image. The FSSVM model is used to train the specific pixels in some positions and find the embedding positions of watermark in carrier image, which is constructed in detail as follows.

Step 1. Select $k$ coordinates from $M \times K$ embedding position coordinates $\left(x_{i}, y_{i}\right)$ at random, where $i=1,2, \ldots, k$. The pixel values corresponding to the original carrier image $I$ are denoted by $I\left(x_{i}, y_{i}\right)$, where $I=\left\{I\left(x_{i}, y_{i}\right) \mid i=1,2, \ldots, k\right\}$.

Step 2. Take each position coordinate $\left(x_{i}, y_{i}\right)$ as the center of the original carrier image $I$ for each selected watermark position $\left(x_{i}, y_{i}\right)$, select an image block with the size $3 \times 3$, and then receive $k$ image blocks.

Step 3. Calculate the corresponding eigenvalues of the image block in the carrier image for each position coordinate $\left(x_{i}, y_{i}\right)$. Namely, the mean value of pixels except the central point $\left(x_{i}, y_{i}\right)$ is calculated as follows:

$$
X\left(x_{i}, y_{i}\right)=\frac{1}{8}\left(\sum_{m=-1}^{1} \sum_{l=-1}^{1} I\left(x_{i}+l, y_{i}+m\right)-I\left(x_{i}, y_{i}\right)\right) .
$$

And the pixel variance except the central point $\left(x_{i}, y_{i}\right)$ is calculated as follows:

$$
\begin{gathered}
D\left(x_{i}, y_{i}\right)=\frac{1}{8}\left(\sum_{m=-1}^{1} \sum_{l=-1}^{1}\left(I\left(x_{i}+l, y_{i}+m\right)-X\left(x_{i}, y_{i}\right)\right)^{2}\right. \\
\left.-\left(I\left(x_{i}, y_{i}\right)-X\left(x_{i}, y_{i}\right)\right)^{2}\right) .
\end{gathered}
$$

Thus, one can totally get $k$ groups of eigenvalues $\left.\left\{X\left(x_{i}, y_{i}\right), D\left(x_{i}, y_{i}\right)\right\}\right|_{i=1,2, \ldots, k}$.

Step 4. Let each feature vector $\left\{X\left(x_{i}, y_{i}\right), D\left(x_{i}, y_{i}\right)\right\}$ be a training data set and the corresponding pixel value $I\left(x_{i}, y_{i}\right)$ of the original carrier image the target value of the training. It constitutes $k$ couples of training sample sets, shown as 


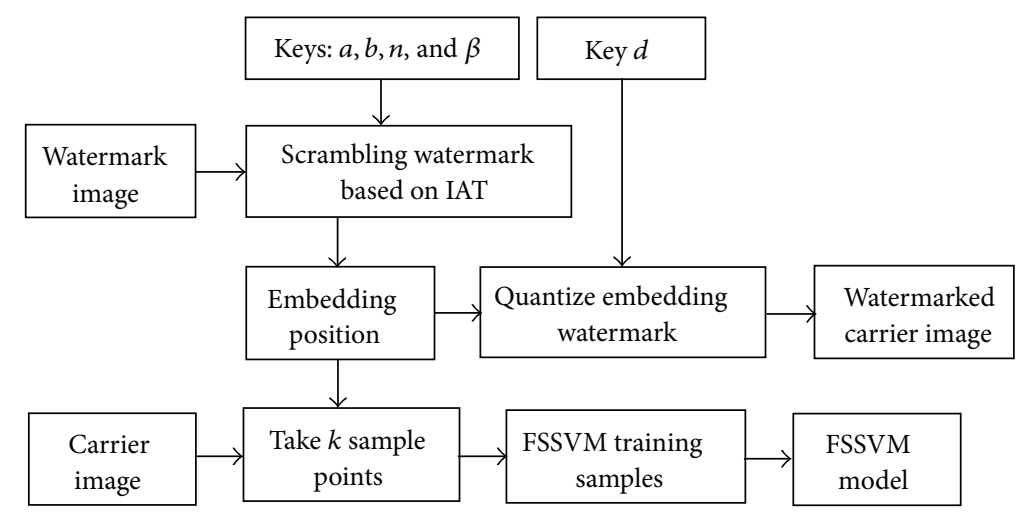

Figure 1: Process of watermark embedding.

$\left.\left\{\left\{X\left(x_{i}, y_{i}\right), D\left(x_{i}, y_{i}\right)\right\} \rightarrow I\left(x_{i}, y_{i}\right)\right\}\right|_{i=1,2, \ldots, k}$, to present a FSSVM training process for the above sample sets as follows.

Step 4.1. Introduce fuzzy membership degree $\mu_{i}$ to each training sample, and blur the input sample set $\left\{\left\{X\left(x_{i}, y_{i}\right)\right.\right.$, $\left.\left.D\left(x_{i}, y_{i}\right)\right\} \rightarrow I\left(x_{i}, y_{i}\right)\right\}$. Fuzzy membership degree $\mu_{i}$ is determined by the relationship between sample input sets $\left\{X\left(x_{i}, y_{i}\right), D\left(x_{i}, y_{i}\right)\right\}$ and optimization values $I\left(x_{i}, y_{i}\right)$.

Step 4.2. Divide the sets into two categories through fuzzy $C$ means clustering to all eigenvalue sets $\left\{X\left(x_{i}, y_{i}\right), D\left(x_{i}, y_{i}\right)\right\}$, find out the center of the two classes, and then calculate the distance $d_{i}$ from the feature vector $\left\{X\left(x_{i}, y_{i}\right), D\left(x_{i}, y_{i}\right)\right\}$ to the center of the corresponding class. Then the membership degree can be expressed as

$$
\mu_{i}=\frac{d_{i}}{d_{\max }},
$$

where $d_{\max }$ represents the maximum distance from feature point to class center for the class of feature vector $\left\{X\left(x_{i}, y_{i}\right), D\left(x_{i}, y_{i}\right)\right\}$.

Step 4.3. Introduce the kernel function $K(x, y)$ to map the sample points into high-dimensional feature space.

Step 4.4. Carry out linear regression in high-dimensional feature space, and obtain the nonlinear regression effect in the original space. Then its regressive function $f$ can be expressed as

$$
f\left(x, x_{i}\right)=\sum_{i=1}^{k}\left(\alpha_{i}-\widehat{\alpha}_{i}\right) K\left(x, x_{i}\right)+\beta .
$$

Here, the kernel function mainly uses Gaussian radial basis function as follows:

$$
\begin{aligned}
& K\left(x, x_{i}\right)=\exp \left(-\frac{\left\|x-x_{i}\right\|^{2}}{\sigma^{2}}\right), \\
& K\left(x, x_{i}\right)=\Phi(x) \cdot \Phi\left(x_{i}\right),
\end{aligned}
$$

where $x, x_{i} \in R_{n}$ represent the input vector $\left\{X\left(x_{i}, y_{i}\right)\right.$, $\left.D\left(x_{i}, y_{i}\right)\right\}, \alpha_{i}$ and $\widehat{\alpha_{i}}$ are the weight coefficients after training, $\beta \in R$ is the deviation, $\Phi$ is a nonlinearity function, and • denotes the inner product operation.

Step 5. Use the principle of structural risk minimization to determine the parameters $\left(\alpha_{i}, \widehat{\alpha_{i}}\right.$, and $\left.\beta\right)$. Namely, transform the original regression equation into solving unconstrained programming problem. Then, the objective function is denoted by

$$
\begin{aligned}
\min & \frac{1}{2}\left(\sum_{i=1}^{k} \alpha_{i}^{2}+\beta^{2}\right) \\
& +\frac{C \mu_{i}}{2} \sum_{i=1}^{k} P\left(\left(1-I_{i}\left(\sum_{i=1}^{k} I_{i} \alpha_{i} \Phi+\beta\right)\right), \lambda\right),
\end{aligned}
$$

where $P(\Delta, \lambda)=\Delta+(1 / \lambda) \ln \left(1+e^{-\lambda \Delta}\right), I_{i}=I\left(x_{i}, y_{i}\right)$ simply, $\lambda$ is an adjustable parameter, and $C>0$ is a constant which is used to determine the complexity of the model and the folded moderation of empirical risk.

3.2. Watermark Embedding Algorithm. The watermark embedding procedure, participating in the optimization flow chart of Figure 1, consists of the processing modules depicted as follows.

Step 1. Input a carrier image $I$ with size $N \times N$ and a binary symbol image $W$ with size $M \times K$. As the original carrier image and the watermark image, they are, respectively, described as $I=\{I(i, j) \mid 1 \leq i \leq N, 1 \leq j \leq N\}, W=\{w(i, j) \mid 1 \leq$ $i \leq M, 1 \leq j \leq K\}$, where $M$ and $K$ are the height and width of binary symbol image, respectively, $I(i, j)$ is the pixel value of the position $(i, j)$ in carrier image, and $w(i, j)$ is the pixel value of the position $(i, j)$ in watermark image.

Step 2. Expand the pixel matrix of binary image into square matrix for making $M=K$, and satisfy the conditions of AT. That is, if $M<K$, then $w(i, j)=1$, where $M<i \leq K$, and if $M>K$, then $w(i, j)=1$, where $K<j \leq M$. Through adding 1 to pixel matrix of binary symbol image $W$, it is expanded into square matrix; that is, $M=K$. AT algorithm can be improved by adding the number of variables and expanding transformation space. Then, the coordinate 
$\left(x_{0}, y_{0}\right)$ of the watermark is taken as initial value to do $n$ times Arnold iteration transformation in binary symbol image. On the basis of such an above consideration, IAT can be denoted by

$$
\left[\begin{array}{l}
x_{n} \\
y_{n}
\end{array}\right]=\left(\left(\left[\begin{array}{cc}
1 & a \\
b & a b+1
\end{array}\right]\left[\begin{array}{l}
x_{0} \\
y_{0}
\end{array}\right]\right) \bmod M\right) \beta
$$

and it follows that

$$
\begin{aligned}
& x_{n}=\left(\left(x_{0}+a y_{0}\right) \bmod M\right) \beta, \\
& y_{n}=\left(\left(b x_{0}+(a b+1) y_{0}\right) \bmod M\right) \beta,
\end{aligned}
$$

where $1 \leq x_{0} \leq M, 1 \leq y_{0} \leq K, 1 \leq x_{n} \leq N, 1 \leq$ $y_{n} \leq N, \beta=$ floor $(N / M)$, floor is down to an integral function, $M$ is the current order of watermark image matrix, $N$ is the order of original carrier image, $a, b$, and $n$ are all positive integers, and $0<n<M^{2} / 2$. Suppose that (11) are taken $n$ times iteration; then the corresponding watermark embedding position $\left(x_{n}, y_{n}\right)$ is got. So $M \times K$ position coordinates $\left(x_{i}, y_{i}\right)$ can be obtained easily, where $i=$ $1,2, \ldots, M \times K$.

In the above conversion formulas, independent parameters $a, b$, and $n$ are defined by the copyright owners themselves, and the three secret key parameters can be used to recover the watermark. $n$ is the times of iteration, which generally ranges from 1 to 20 . Here, if $n$ is too large, it will affect the running speed of the program and increase the timespace complexity of IAT. $\beta$ is the enlargement factor to the value of $\left(x_{0}, y_{0}\right)$ after $n$ times iteration transformation. When the watermark is embedded, one needs to make $\left(x_{n}, y_{n}\right)$ adapt to the size of the whole carrier image instead of focusing on a certain square. Namely, all of the embedding positions are dispersed into the whole original carrier image.

Step 3. Let $M \times K$ position coordinates be the embedding positions of watermark in the carrier image. That is, embed the pixel of the watermark position $\left(x_{0}, y_{0}\right)$ into the position $\left(x_{n}, y_{n}\right)$ of carrier image after getting $M \times K$ embedding positions $\left(x_{i}, y_{i}\right)$, where $i=1,2, \ldots, M \times K$. Then select $k$ position coordinates $\left(x_{i}, y_{i}\right)$ at random from embedding positions to do FSSVM training, where $i=1,2, \ldots, k$.

Step 4. Determine the pixel value $I^{\prime}\left(x_{n}, y_{n}\right)$ of the embedded position in the original carrier image by using quantitative embedding rules. $d=10$ is used as the quantifying step size of the pixel value $I\left(x_{n}, y_{n}\right)$, and $k=\operatorname{round}\left(I\left(x_{n}, y_{n}\right) / d\right)$, where round is rounded to the nearest integral function. Then, to determine the pixel value $I^{\prime}\left(x_{n}, y_{n}\right)$, the several cases are given as follows.

(1) If the watermark pixel value $w\left(x_{0}, y_{0}\right)=1$ and $k=$ $2 m+1$ hold, where $m \in N, k$ is an odd number, then one has that

$$
I^{\prime}\left(x_{n}, y_{n}\right)=\left(\operatorname{round} \frac{I\left(x_{n}, y_{n}\right)}{d}\right) d+5
$$

(2) If the watermark pixel value $w\left(x_{0}, y_{0}\right)=0$ and $k=$ $2 m+1$ hold, where $m \in N, k$ is an odd number, then one has that

$$
I^{\prime}\left(x_{n}, y_{n}\right)=\left(\operatorname{round} \frac{I\left(x_{n}, y_{n}\right)}{d}\right) d+5
$$

(3) If the watermark pixel value $w\left(x_{0}, y_{0}\right)=1$ and $k=2 m$ hold, where $m \in N, k$ is an even number, then one has that

$$
I^{\prime}\left(x_{n}, y_{n}\right)=\left|\left(\operatorname{round} \frac{I\left(x_{n}, y_{n}\right)}{d}\right) d-5\right|
$$

(4) If the watermark pixel value $w\left(x_{0}, y_{0}\right)=0$ and $k=2 m$ hold, where $m \in N, k$ is an even number, then one has that

$$
I^{\prime}\left(x_{n}, y_{n}\right)=\left|\left(\operatorname{round} \frac{I\left(x_{n}, y_{n}\right)}{d}\right) d-5\right|
$$

Here, compared with the traditional embedding rules, the magnitude of pixel value in the original carrier image, which is changed by quantitative rule, is relatively small, and the biggest changed value is 10 . Thus it can realize the imperceptibility after embedding the watermark. According to the above four rules, the pixel values of $M \times K$ embedding coordinate positions $\left(x_{n}, y_{n}\right)$ of watermark can be calculated in the watermarked carrier image easily.

Step 5. Replace the original pixel values in the carrier image with those of the calculated $M \times K$ embedding coordinate positions $\left(x_{n}, y_{n}\right)$; namely, $\left.I\left(x_{n}, y_{n}\right) \rightarrow I^{\prime}\left(x_{n}, y_{n}\right)\right|_{i=1,2, \ldots, M \times K}$. Then, the watermarked carrier image can be obtained, whose size is still $N \times N$, and only the pixel values of a minority of points change slightly.

3.3. Watermark Extraction Algorithm. The watermark extraction procedures are illustrated with the help of block diagrams in Figure 2. The watermark extraction process can be summarized as follows.

Step 1. Denote the watermarked carrier image by $I^{\prime}$, whose size is $N \times N . I^{\prime}(i, j)$ represents the pixel value of the position $(i, j)$ in watermarked carrier image, where $I^{\prime}=\left\{I^{\prime}(i, j) \mid 1 \leq\right.$ $i \leq N, 1 \leq j \leq N\}$.

Step 2. Test $I^{\prime}$ using FSSVM model obtained from the process of watermark embedding as follows. Firstly, calculate the eigenvalues of each pixel in $\left(x_{i}, y_{i}\right)$ of $I^{\prime}$, where the mean pixel value of eight points except the central point $\left(x_{i}, y_{i}\right)$ is calculated by

$$
X\left(x_{i}, y_{i}\right)=\frac{1}{8}\left(\sum_{m=-1}^{1} \sum_{l=-1}^{1} I^{\prime}\left(x_{i}+l, y_{i}+m\right)-I^{\prime}\left(x_{i}, y_{i}\right)\right) .
$$




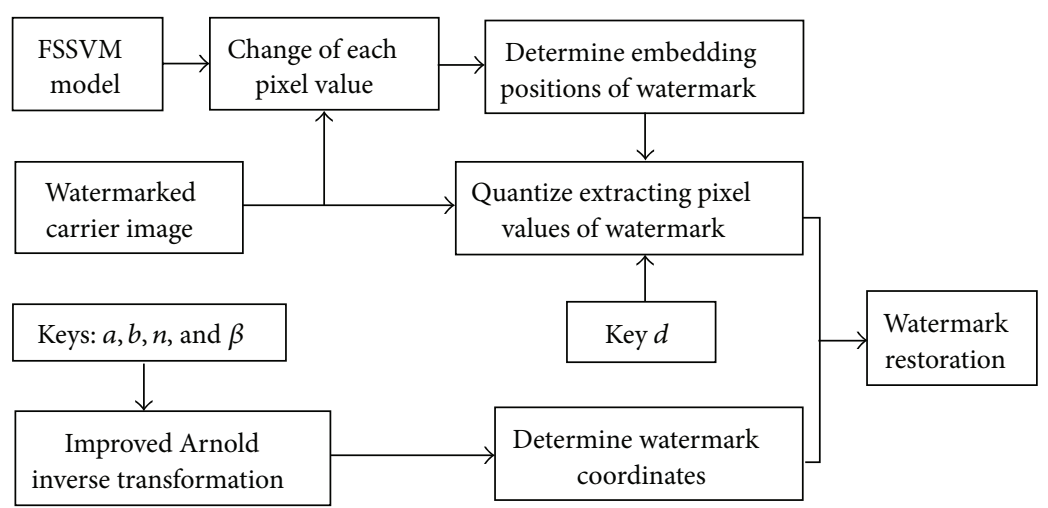

Figure 2: Process of watermark extraction.

And the pixel variance of eight points except the central point $\left(x_{i}, y_{i}\right)$ is calculated by

$$
\begin{gathered}
D\left(x_{i}, y_{i}\right)=\frac{1}{8}\left(\sum_{m=-1}^{1} \sum_{l=-1}^{1}\left(I^{\prime}\left(x_{i}+l, y_{i}+m\right)-X\left(x_{i}, y_{i}\right)\right)^{2}\right. \\
\left.-\left(I^{\prime}\left(x_{i}, y_{i}\right)-X\left(x_{i}, y_{i}\right)\right)^{2}\right) .
\end{gathered}
$$

Then, $N \times N$ groups of eigenvalues $\left\{X\left(x_{i}, y_{i}\right)\right.$, $\left.D\left(x_{i}, y_{i}\right)\right\}\left.\right|_{i=1,2, \ldots, N \times N}$ can be obtained totally. Let the $N \times N$ groups of eigenvalues $\left.\left\{X\left(x_{i}, y_{i}\right), D\left(x_{i}, y_{i}\right)\right\}\right|_{i=1,2, \ldots, N \times N}$ be the input data sets $x$ and $y$ of FSSVM model test. Namely, $X\left(x_{i}, y_{i}\right)$ and $D\left(x_{i}, y_{i}\right)$ substitute $x$ and $y$ into the model, respectively, and the result is calculated by using the following regressive function:

$$
f(x, y)=\sum_{i=1}^{k}\left(\alpha_{i}-\widehat{\alpha}_{i}\right) K(x, y)+\beta
$$

In (18), $\alpha_{i}, \widehat{\alpha}_{i}$, and $\beta$ have been derived from the training in the process of watermark embedding. So the predicted pixel values $I_{0}^{\prime}(i, j)$ of $N \times N$ positions in the watermarked carrier image can be got easily.

The eigenvalue selection refers to one-order moment and second-order moment of the probability statistics. In fact, one can also increase the type of eigenvalue, such as the thirdorder moment, which is beneficial for improving the prediction accuracy. Here, the $n$-order moment $A$ can be briefly expressed as

$$
A=E\left(\sum_{i, j}\left(I^{\prime}(i, j)-\bar{X}\right)^{n}\right)
$$

where $\bar{X}$ represents the average values of pixel points except the central point in selected image blocks and $E$ is to solve the average.
Step 3. Compare the pixel values $I^{\prime}(i, j)$ of $I^{\prime}$ with the predictive pixel values $I_{0}^{\prime}(i, j)$ of the $N \times N$ positions, and calculate their difference matrix $D$, which is denoted by

$$
D=\left|I^{\prime}(i, j)-I_{0}^{\prime}(i, j)\right|
$$

where $1 \leq i \leq N, 1 \leq j \leq N$. Then sort each element and take out the top $M \times K$ pixel values corresponding to $D$, which are progressively becoming smaller. Their corresponding position coordinates $(i, j)$ in carrier image, which are embedded with watermark, are obtained. Process the $M \times$ $K$ pixel coordinates $(i, j)$ by improving Arnold inverse transformation. Namely, take the pixel coordinate $(i, j)$ as initial value to do $n$ times iteration. Thus, the corresponding position coordinate $\left(x_{0}, y_{0}\right)$ for $(i, j)$ in the watermark image can be got from the following formula:

$$
\left[\begin{array}{l}
x_{0} \\
y_{0}
\end{array}\right]=\left(\left[\begin{array}{cc}
1 & a \\
b & a b+1
\end{array}\right]^{-1}\left[\begin{array}{c}
\frac{i}{\beta} \\
\frac{j}{\beta}
\end{array}\right]\right) \bmod M
$$

and it follows that

$$
\begin{aligned}
& x_{0}=\left((a b+1) \frac{i}{\beta}-b \frac{j}{\beta}\right) \bmod M, \\
& y_{0}=\left(-a \frac{i}{\beta}+\frac{j}{\beta}\right) \bmod M,
\end{aligned}
$$

where $1 \leq x_{0} \leq M, 1 \leq y_{0} \leq K, M=K, \beta=$ floor $(N / M)$, -1 describes the inverse matrix, $M$ is the order of watermark matrix, $N$ is the order of carrier image, $a, b$, and $n$ are all positive integers, and $0<n<M^{2} / 2$. Suppose that, in every iteration, $i / \beta$ is used by the assignment of $x_{0}, j / \beta$ is used by the assignment of $y_{0}$, and (22) are taken $n$ times iteration, and then the position coordinates $\left(x_{0}, y_{0}\right)$ of watermark corresponding to $(i, j)$ can be obtained. Finally, $M \times K$ position coordinates $\left(x_{i}, y_{i}\right)$ can be obtained easily, where $i=1,2, \ldots, M \times K$, which are the coordinate values of the original watermark.

Step 4. Compute the corresponding pixel values in the watermark, when the position coordinates $(i, j)$ of the watermarked 


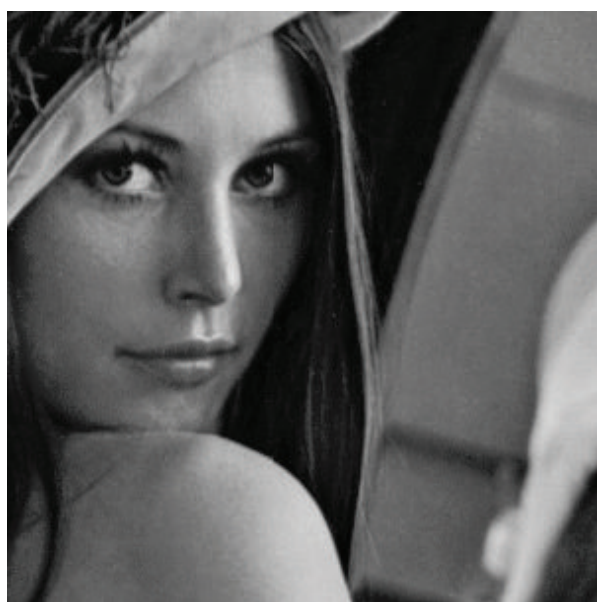

(a)

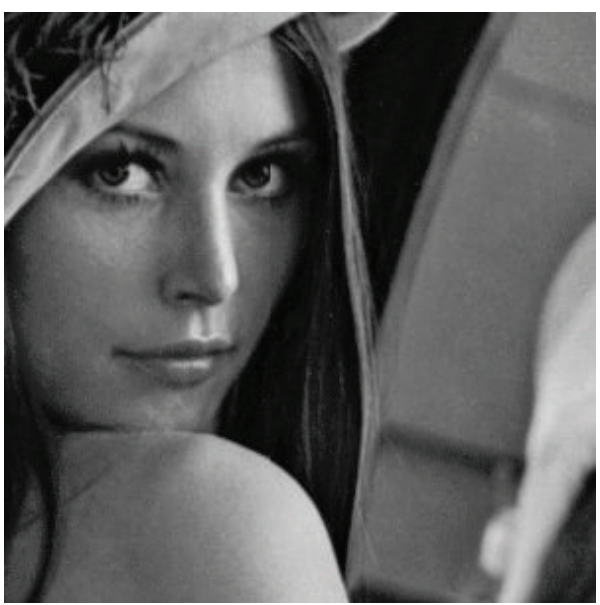

(c)

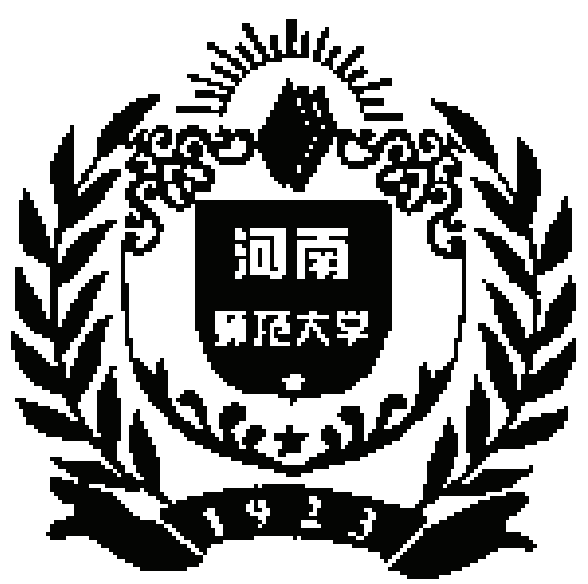

(b)

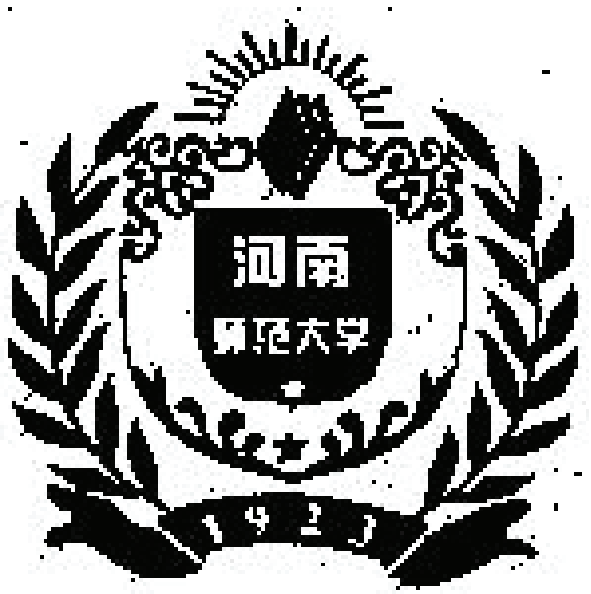

(d)

FIgURE 3: (a) Original carrier image; (b) original watermark image; (c) the watermarked image; (d) the extracted watermark without attack.

carrier image have been determined and their corresponding position coordinates $\left(x_{0}, y_{0}\right)$ in the watermark have also been calculated. Namely, according to the change rules of the pixel values in the watermarked carrier image, the pixel recovery rules of the current watermark are deduced in the process of watermark extraction.

Suppose that $\lambda=\operatorname{floor}\left(I^{\prime}(i, j) / d\right)$; then $d=10$ is still the size of quantifying step of pixel value, and $I^{\prime}(i, j)$ is the pixel value at the position $(i, j)$ of the watermarked carrier image. If $\lambda=2 m+1$, where $m \in N$, then the $\lambda$ is an odd number, and $w(i, j)=1$. It follows that the pixel value of watermark is 1 . If $\lambda=2 m$, where $m \in N$, then the $\lambda$ is an even number, and $w(i, j)=0$. It follows that the pixel value of watermark is 0 . However, the current position coordinate $(i, j)$ is not the position coordinate corresponding to the watermark. The recovery rules of the pixel values just embody the advantages of binary watermark image, because the pixel value is either 0 or 1 . So it does not depend on the pixel characteristics, and the pixel values of watermark can be judged only by the quantitative extraction rules of the pixel value in the watermarked carrier image.
Step 5. Restore the pixel values of original watermark image according to the values of $w(i, j)$, which are calculated at the position of $M \times K$ pixel coordinates and selected from $I^{\prime}$. Then, each position coordinate $(i, j)$ is replaced by $\left(x_{0}, y_{0}\right)$ through improved Arnold inverse transformation; that is, $w(i, j) \rightarrow w\left(x_{0}, y_{0}\right)$. The coordinate values of watermark and the corresponding pixel values have been determined. According to the above two results, the original watermark image $W$ can be recombined.

\section{Experimental Works}

For the experimental purposes, specific software has been developed to implement the proposed methods using MATLAB. Figure 3(a) shows an original carrier image with size $300 \times 300$, which is Lena gray image of unit 8 . Figure $3(b)$ depicts an original watermark image with size $150 \times 150$, which is the school badge image of Henan Normal University with binary sequence. When the watermark is embedded, the positions embedded into the carrier image are determined by IAT, and the secret key parameters are set as $a=1, b=2$, 
$n=1$, and $\beta=2$. Then, the pixel values of the embedding positions are determined. When the watermark is extracted, the embedded positions should be found out by using FSSVM model, and the pixel values are restored. Thus, the embedding position is transformed inversely back to the original watermark by using improved Arnold inverse transformation. The above two processes can complement each other. Figure 3(c) is the Lena digital image embedded with actual watermark image by using the watermark embedding algorithm in this paper.

The quality and perceptibility of digital image embedded with actual watermark are judged by using the value of peak signal-to-noise ratio (PSNR), which presents the damaged degree of embedded watermark information to carrier quality. The bigger the PSNR is, the smaller the damaged degree is. Then, PSNR is denoted by

$$
\text { PSNR }=10 \log _{10} \frac{m n \max _{m, n}\left(\left(I_{i, j}\right)^{2}\right)}{\sum_{i=1}^{m} \sum_{j=1}^{n}\left(I_{i, j}-I_{i, j}^{\prime}\right)^{2}},
$$

where $m=300, n=300$, and $I_{i, j}$ and $I_{i, j}^{\prime}$ describe the pixel values of the original carrier image and the watermarked carrier image, respectively.

The bit error rate (BER) between extracted watermark and original watermark is employed to evaluate the extracted watermark image objectively. The closer the distance between BER and 0 is, the higher the robustness of the watermark system is, and the stronger the antiattack capability is. Then, BER is denoted by

$$
\mathrm{BER}=\frac{\sum_{i=1}^{M} \sum_{j=1}^{K} w(i, j) \oplus w^{\prime}(i, j)}{M K},
$$

where $M=150, K=150, w(i, j)$ and $w^{\prime}(i, j)$ describe the pixel values of the original watermark and the extracted watermark in corresponding positions, respectively, and $\oplus$ represents XOR operation by bit.

The objective evaluation of the test result of watermark can also use normalized correlation coefficient (NCC) to evaluate the similarity degree, which describes the change before and after embedding watermark in the carrier image. The greater the similarity degree is, the higher the robustness of watermark is. Then, NCC is denoted by

$$
\mathrm{NCC}=\frac{\sum_{m, n} I_{m, n} I_{m, n}^{\prime}}{\sum_{m, n}\left(I_{m, n}\right)^{2}}
$$

It follows from Figure 3(c) that PSNR is very high and gets $36.3697 \mathrm{~dB}$, and the quality of the watermarked image has no change, which is consistent with Figure 3(a). Hence, it fully satisfies the requirement of imperceptibility of watermark. In what follows, the watermark shown in Figure 3(d) is extracted from Figure 3(c) by using the proposed extraction algorithm. When the watermarked image has not got any attack, the actual watermark can almost be extracted losslessly, BER = 0.0059 is approximately equal to 0 , and NCC $=0.9785$ is very close to 1 . Therefore, the extracted watermark is almost the same as the original watermark.
TABLE 1: Experimental results of watermark imperceptibility and robustness.

\begin{tabular}{lcc}
\hline Attacks & PSNR & BER \\
\hline Image brightening $(+75)$ & 21.6031 & 0.0124 \\
Image darkening $(-50)$ & 12.5791 & 0.0074 \\
Image histogram equalization & 15.9505 & 0.0088 \\
Gaussian noise $(\mu=0$ and $\sigma=0.02)$ & 23.9825 & 0.0730 \\
Median filter $([9 \times 9])$ & 31.5116 & 0.0270 \\
JPEG compression $(10 \%)$ & 22.7442 & 0.0737 \\
Geometric cutting $($ left $100 \times 300)$ & 12.5184 & 0.0126 \\
Geometric rotation $10^{\circ}$ & 22.0357 & 0.0142 \\
\hline
\end{tabular}

In order to further investigate the robustness of our proposed scheme, the watermarked image shown in Figure 3(c) is distorted by taking various attacks, respectively, like (1) image brightening, (2) image darkening, (3) histogram equalization, (4) superposition of Gaussian noise, (5) median filter, (6) JPEG compression, (7) geometric cutting, and (8) geometric rotation. Then, Table 1 shows the experimental data under the above eight types of attacks, and Figures $4(\mathrm{a})-4(\mathrm{~h})$ display the watermarks extracted from the watermarked images which are manipulated by the above attack operations, respectively. From Table 1, the results show that the actual embedded watermarks still have ideal capability of antiattacks, and the BER values are equal to 0 approximately. It follows from Figure 4 that these embedded watermarks can be extracted well and are close to the result of no attacks. Thus, the proposed method in this paper has better robustness against various attacks.

The following part of our experiments continues testing the proposed algorithms in a new watermark image with size $35 \times 35$, shown in Figure 5(a), while the carrier image is still Figure 3(a). By using the embedding algorithm in this paper, the quality of the watermarked image has not changed too much, and PSNR is very high, up to $50.0612 \mathrm{~dB}$, which is consistent with Figure 3(a). Then, it fully satisfies the requirements of the watermark invisibility. Figure 5(b) is the watermark extracted by using the proposed extraction algorithm. It shows that when the watermarked image has not suffered any attack processing, the embedded watermark can be extracted losslessly, BER $=0.0049$ is approximate to 0 , and NCC $=0.9956$ is very close to 1 . So the extracted watermark is almost the same as Figure 5(a). What is more, the watermarked image further suffers the above eight types of attacks, and then the corresponding extracted watermark images are shown in Figures 5(c)-5(j). With respect to PSNR and BER, our proposed scheme (IAT + FSSVM) is compared with the other two methods, including the fact that IAT is replaced by AT to combine AT and FSSVM (AT + FSSVM) method and FSSVM is replaced by Least Squares Support Vector Machine (LSSVM) to combine IAT and LSSVM (IAT + LSSVM) method. The experimental results are illustrated in Table 2.

The third part of our experiments is to test the proposed algorithms in a camera man image with size $300 \times 300$, shown in Figure 6, while the watermark is still Figure 3(b). 


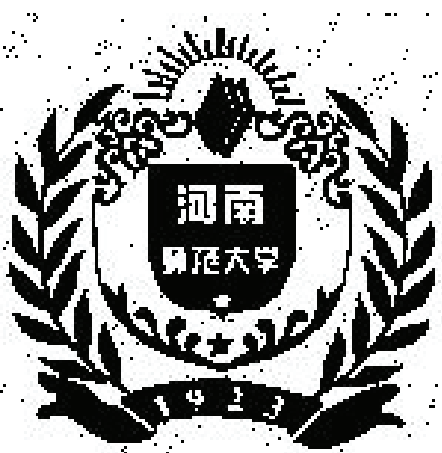

(a)

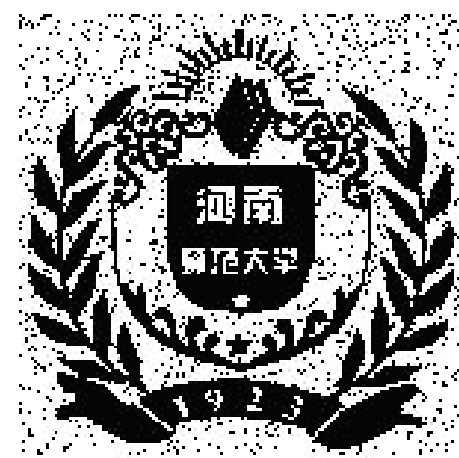

(d)

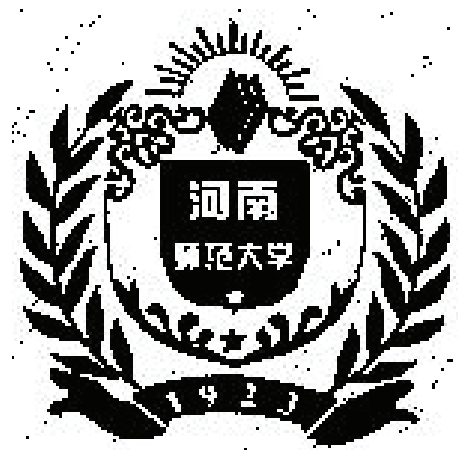

(b)

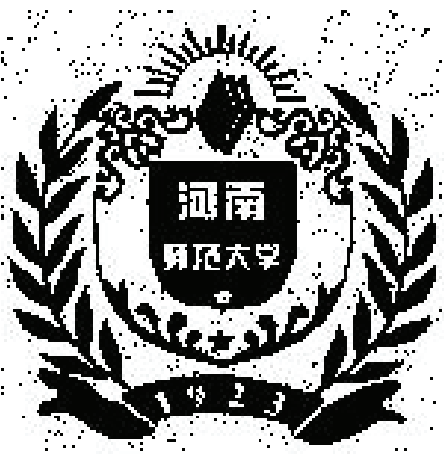

(e)

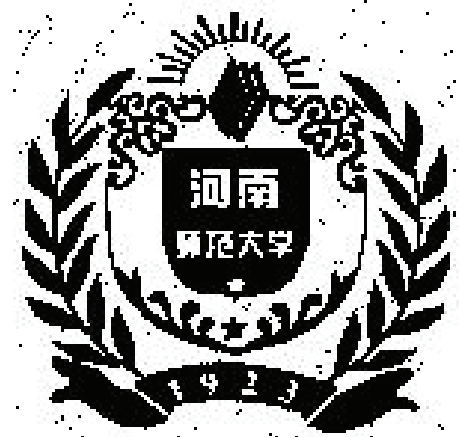

(c)

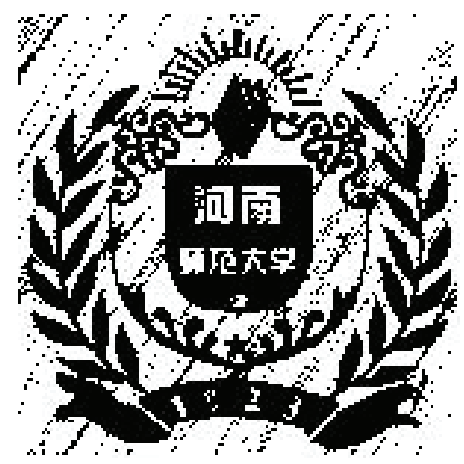

(f)

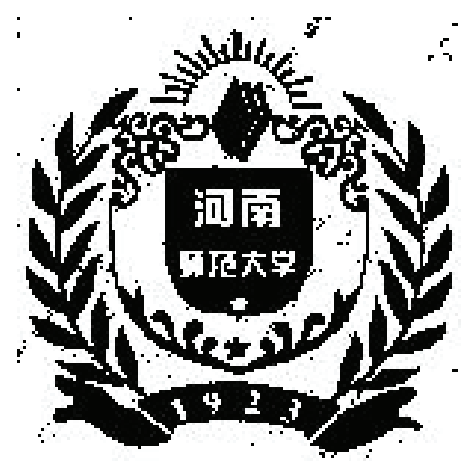

(g)

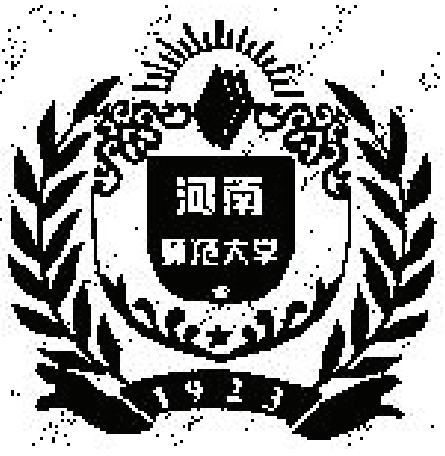

(h)

Figure 4: (a) The extracted watermark after brightening (+75); (b) the extracted watermark after darkening $(-50)$; (c) the extracted watermark after equalization; (d) the extracted watermark after adding Gaussian noise; (e) the extracted watermark after median filter; (f) the extracted watermark after compression; (g) the extracted watermark after cutting; (h) the extracted watermark after rotation.

The camera man image is embedded with Figure 3(b) by using the proposed embedding algorithm, and then PSNR is very high, up to $73.9578 \mathrm{~dB}$. Figure $7(\mathrm{a})$ is the watermark extracted from the watermarked camera man image by using the proposed extraction algorithm. When the watermarked camera man image has not suffered any attack processing, the embedded actual watermark can be almost extracted losslessly, $\mathrm{BER}=0.0035$ is approximate to 0 , and $\mathrm{NCC}=1$. Hence, Figure 7(a) is almost the same as Figure 3(b). In what follows, the watermarked camera man image further suffers the above eight attacks, and then the corresponding extracted watermark images are shown in Figures 7(b)-7(i). Similarly, with respect to PSNR and BER, the experimental results of three methods (AT + FSSVM, IAT + LSSVM, and IAT + FSSVM) are shown in Table 3.

From Tables 2 and 3, it can be concluded that the combination of IAT and FSSVM proposed in this paper makes the BER and PSNR very ideal. On the one hand, though the PSNR values between AT + FSSVM and IAT + FSSVM are approximate, the BER values in our scheme are obviously smaller; then it is obvious that IAT has more advantages in the watermark extraction. On the other hand, though the PSNR values between IAT + LSSVM and IAT + FSSVM are identical, half of the BER values in our scheme are slightly smaller and the others are approximate, so it can be concluded that the FSSVM model is more powerful in the watermark 


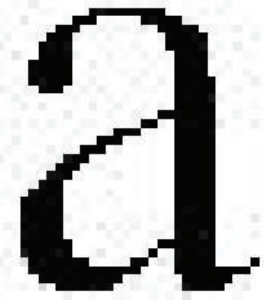

(a)

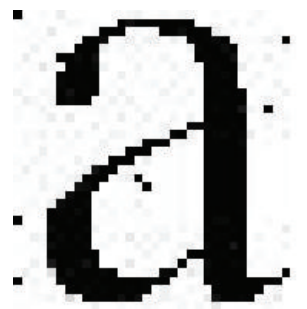

(f)

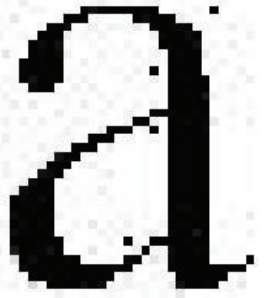

(b)

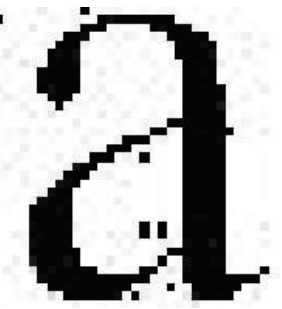

(g)

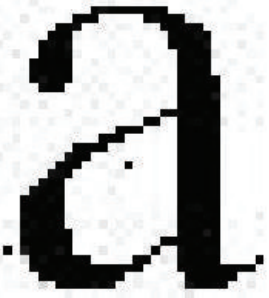

(c)

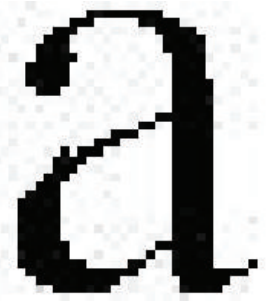

(h)

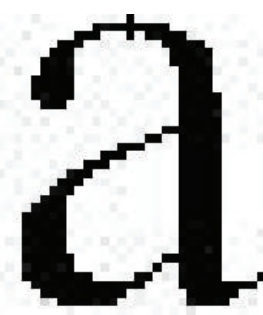

(d)

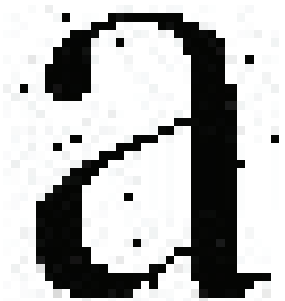

(i)

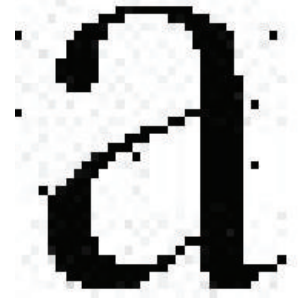

(e)

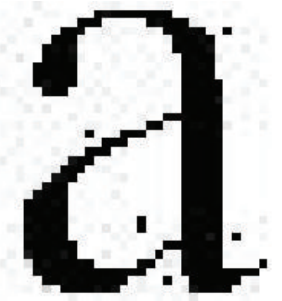

(j)

FIGURE 5: (a) The original changed watermark; (b) the extracted watermark without attack; (c) the extracted watermark after brightening $(+75)$; (d) the extracted watermark after darkening $(-50)$; (e) the extracted watermark after equalization; (f) the extracted watermark after adding Gaussian noise; (g) the extracted watermark after median filter; (h) the extracted watermark after compression; (i) the extracted watermark after cutting; $(\mathrm{j})$ the extracted watermark after rotation.

TABLE 2: Experimental results of watermark imperceptibility and robustness after changing watermark.

\begin{tabular}{|c|c|c|c|c|c|c|}
\hline \multirow{2}{*}{ Attacks } & \multicolumn{2}{|c|}{$\mathrm{AT}+\mathrm{FSSVM}$} & \multicolumn{2}{|c|}{ IAT + LSSVM } & \multicolumn{2}{|c|}{ IAT + FSSVM } \\
\hline & PSNR & BER & PSNR & BER & PSNR & BER \\
\hline Image brightening $(+75)$ & 15.6836 & 0.1706 & 21.5951 & 0.0024 & 21.5951 & 0.0041 \\
\hline Image darkening $(-50)$ & 13.8697 & 0.1739 & 12.9168 & 0.0016 & 12.9168 & 0.0033 \\
\hline Image histogram equalization & 15.9308 & 0.1249 & 15.9258 & 0.0016 & 15.9258 & 0.0065 \\
\hline Gaussian noise $(\mu=0$ and $\sigma=0.02)$ & 24.0350 & 0.4710 & 23.9928 & 0.0155 & 23.9928 & 0.0122 \\
\hline Median filter $([9 \times 9])$ & 31.4799 & 0.4531 & 31.4850 & 0.0155 & 31.4850 & 0.0123 \\
\hline JPEG compression (10\%) & 22.7381 & 0.4702 & 22.7412 & 0.0073 & 22.7412 & 0.0059 \\
\hline Geometric cutting (left $100 \times 300)$ & 31.5740 & 0.3045 & 11.6074 & 0.0049 & 11.6074 & 0.0080 \\
\hline Geometric rotation $10^{\circ}$ & 22.4917 & 0.3086 & 18.8479 & 0.0106 & 18.8479 & 0.0110 \\
\hline
\end{tabular}

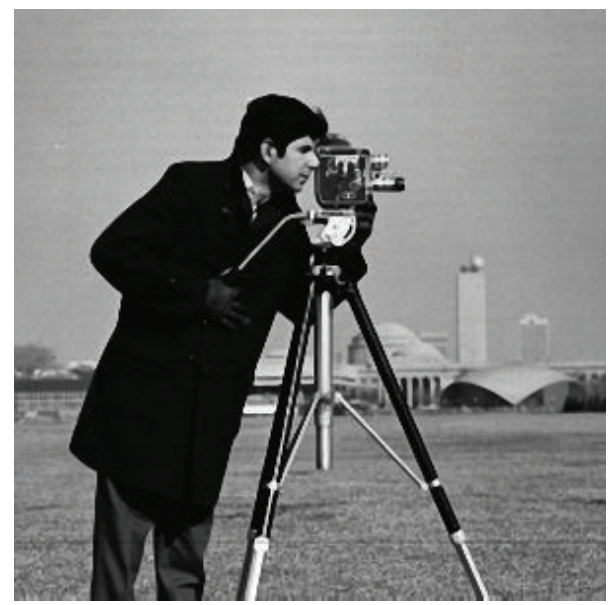

FIgURE 6: The original changed carrier image. extraction. Therefore, the performances of IAT and FSSVM are better than those of AT and LSSVM.

The fourth part of our experiments is to give more details and justifications of our scheme by taking six carrier images against thirteen kinds of attacks. The watermark is still Figure 3(b), and the carrier images include Clock, Aerial, Girl, Truck, Airport, and Man standard images, respectively, which are downloaded from USC-SIPI image library. The thirteen kinds of attack indexes present free, JPEG, low-pass filtering, median filtering, average filtering, salt peppers noise, Gaussian noise, cropping $25 \%$, rotation $15^{\circ}$, sharpening, brightening, darkening, and histogram equalization, respectively. The BER values between original and extracted watermark for the six carrier images against thirteen attacks are shown in Figure 8, which shows that the Airport and Man with size $1024 \times$ 1024 have the least BER, the Clock and Aerial with size $256 \times$ 256 have the most BER, and the sizes of Girl and Truck are 


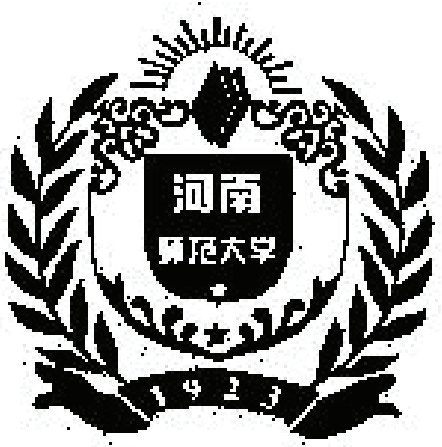

(a)

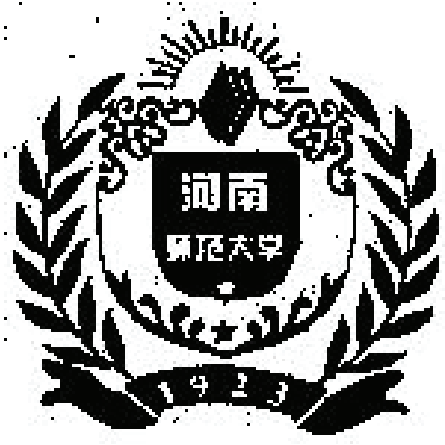

(d)

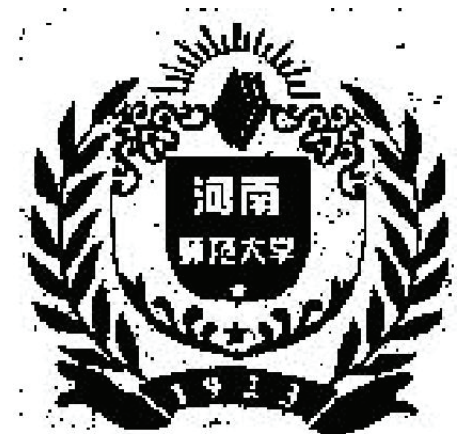

(g)

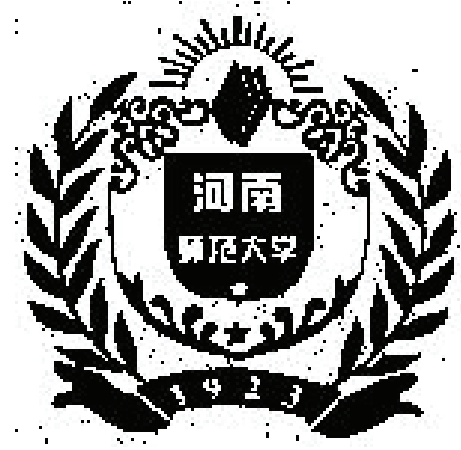

(b)

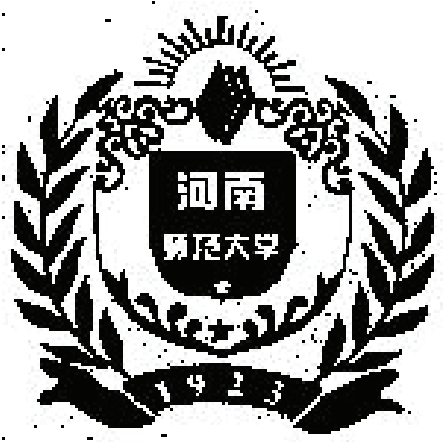

(e)

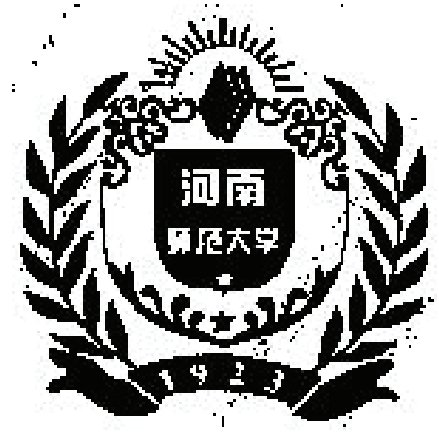

(h)

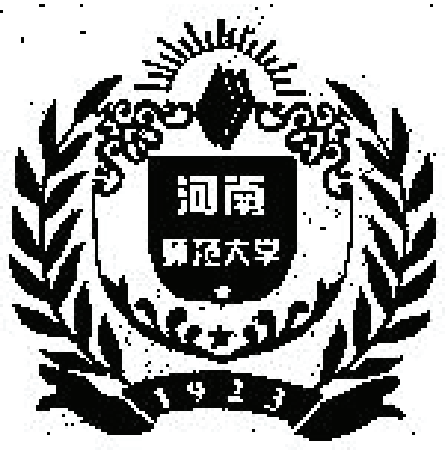

(c)

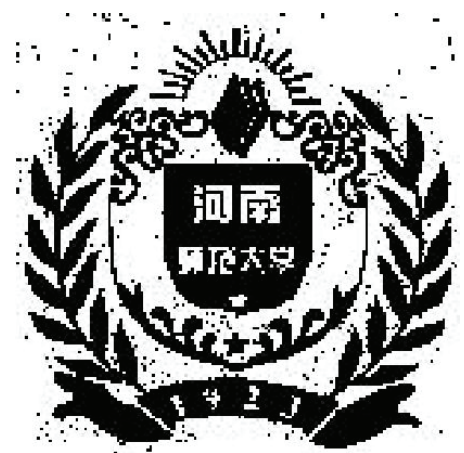

(f)

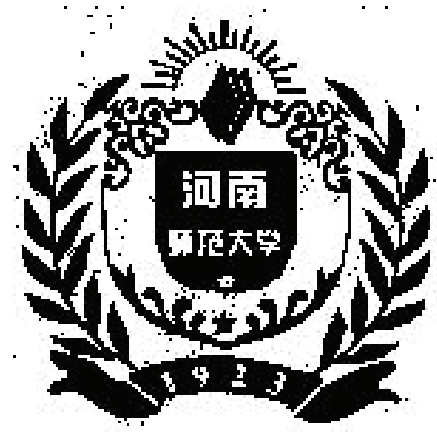

(i)

FIGURE 7: (a) The extracted watermark without attack; (b) the extracted watermark after brightening (+75); (c) the extracted watermark after darkening (-50); (d) the extracted watermark after equalization; (e) the extracted watermark after adding Gaussian noise; (f) the extracted watermark after median filter; (g) the extracted watermark after compression; (h) the extracted watermark after cutting; (i) the extracted watermark after rotation.

TABLE 3: Experimental results of watermark imperceptibility and robustness after changing carrier image.

\begin{tabular}{|c|c|c|c|c|c|c|}
\hline \multirow{2}{*}{ Attacks } & \multicolumn{2}{|c|}{$\mathrm{AT}+\mathrm{FSSVM}$} & \multicolumn{2}{|c|}{ IAT + LSSVM } & \multicolumn{2}{|c|}{ IAT + FSSVM } \\
\hline & PSNR & BER & PSNR & BER & PSNR & BER \\
\hline Image brightening $(+75)$ & 14.8985 & 0.0347 & 15.0029 & 0.0095 & 15.0029 & 0.0091 \\
\hline Image darkening $(-50)$ & 16.5895 & 0.0158 & 16.4844 & 0.0039 & 16.4844 & 0.0084 \\
\hline Image histogram equalization & 21.9937 & 0.0439 & 21.9832 & 0.0101 & 21.9832 & 0.0067 \\
\hline Gaussian noise $(\mu=0$ and $\sigma=0.02)$ & 38.8298 & 0.0431 & 37.8283 & 0.0054 & 37.8283 & 0.0076 \\
\hline Median filter $(9 \times 9)$ & 33.0709 & 0.1410 & 34.5537 & 0.0381 & 34.5537 & 0.0277 \\
\hline JPEG compression (10\%) & 36.1963 & 0.0379 & 34.7436 & 0.0131 & 34.7436 & 0.0135 \\
\hline Geometric cutting (left $100 \times 300)$ & 23.6165 & 0.0193 & 12.8017 & 0.0049 & 12.8017 & 0.0106 \\
\hline Geometric rotation $10^{\circ}$ & 18.8558 & 0.0530 & 22.5508 & 0.0135 & 22.5508 & 0.0073 \\
\hline
\end{tabular}




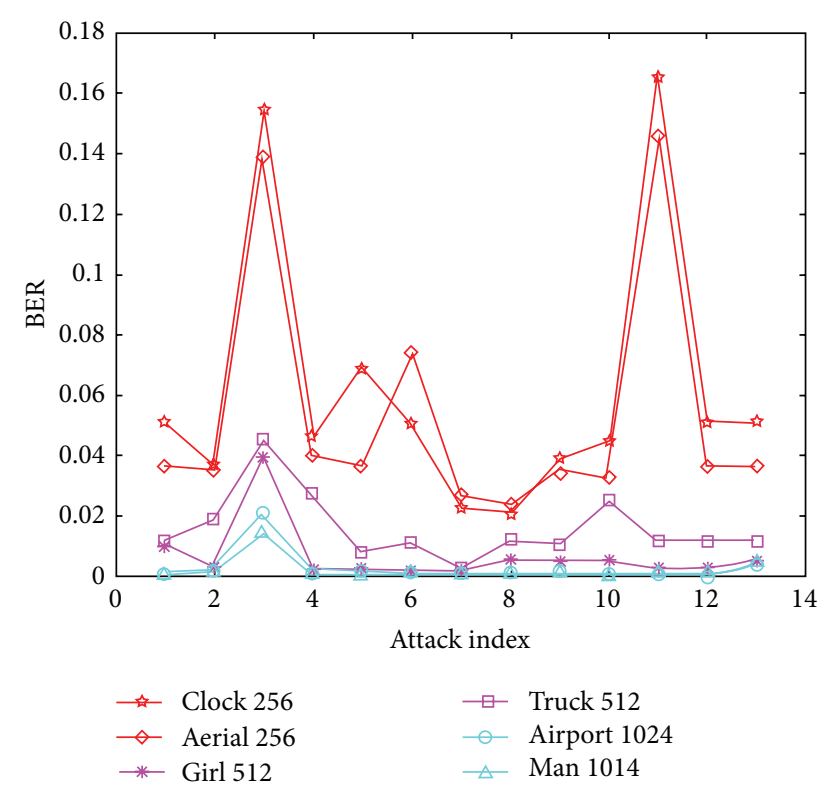

FIgURE 8: The BER for six carrier images against thirteen attacks.

$512 \times 512$. That is, the larger the carrier is, the lesser the BER is. The reason is the parameter $\beta$ of IAT in our scheme, which makes the watermark pixels evenly dispersed in the whole carrier and minimizes the damage of being attacked, while the larger the carrier is, the larger the $\beta$ is, the more the pixels disperse, the less the damage is, and as a result, the lesser the BER is. So the proposed embedding and extraction methods can be considered reasonable and feasible.

The last part of our experiments is to compare our proposed scheme with the other state-of-the-art watermarking methods based on SVM, which are Peng's method [24], Tsai's method [22], and Li's method [23], respectively. The watermark is still Figure 3(b), and the carrier image is Boat with size $512 \times 512$, downloaded from USC-SIPI image library. The top-ten kinds of the above thirteen kinds of attacks are given to the watermarked Boat image. Then the BER values of our scheme compared with the other three methods against ten attacks are shown in Figure 9. It can be concluded from Figure 9 that our scheme outperforms the other three methods as a whole, though the BER values of our scheme are a little bigger than those of others against the low-pass filtering and Gaussian noise.

From the above experimental results, the comparative analysis of our scheme with other schemes and the advantages of our algorithms are further summarized as follows.

(1) The embedding method presented in this paper is realized by using IAT and quantization rules, and more parameters $(a, b, n, \beta$, and $d)$ are used, where $a, b, n$, and $\beta$ are introduced in IAT and $d$ appears in quantization rules. These parameters are used as secret keys to extract watermark in the process of watermark extraction, which can be obtained from the embedding process. Because the number of secret keys increases to five and every key has many possible values, our extraction scheme is very difficult to break; then the security of the proposed embedding and extraction algorithms is

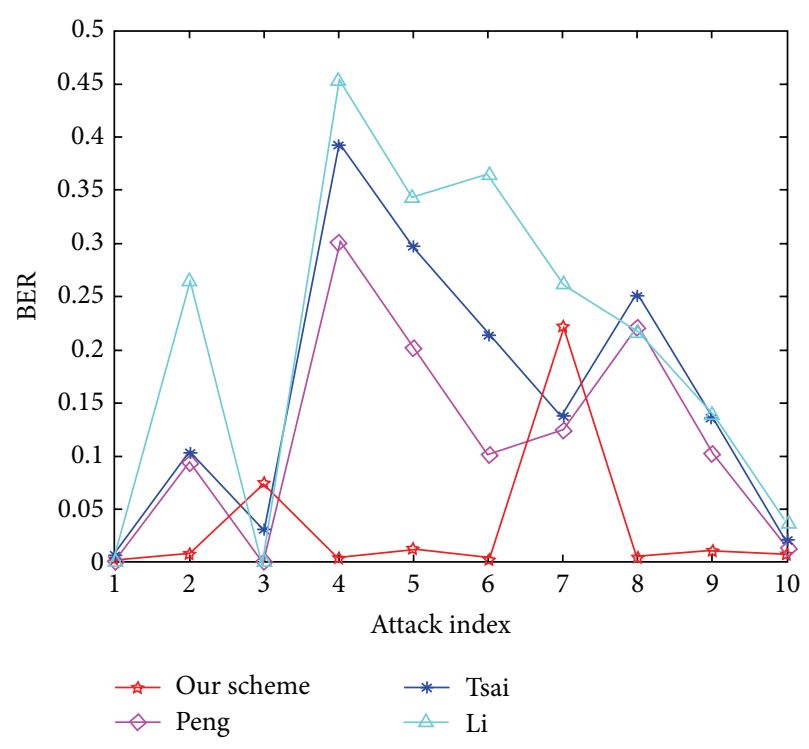

FIGURE 9: The BER of four watermarking schemes for Boat against ten attacks.

improved greatly. Besides, the watermark can be extracted without the original carrier by using our scheme; then the blind extraction is realized, which expands the watermarking applications and improves the security degree. In addition, the change range of pixel values in the carrier image with the quantitative embedding rules is very small. Therefore, compared with the traditional embedding techniques of watermark, the proposed scheme not only realizes the complete imperceptibility of the watermark, but also causes minor damage to the original carrier image. Thus, the problem of image degradation does not exist, so it maintains the integrity of original information of the carrier and the imperceptibility of the carrier image with watermark.

(2) Compared with the existing SVM-based watermarking techniques, the FSSVM model proposed in this paper introduces the concept of fuzzy membership degree and combines the fuzzy mathematics with smooth SVM to simulate the visual features of the human eyes for the watermarked carrier image instead of the standard SVM. The application method not only provides a new solution for digital image watermark technique, but also quickens the training speed and improves the efficiency of image characteristic value. The predicted pixel results of test sample are closer to the actual values than the standard SVM. Thus, the extracted watermark is very close to the original watermark.

(3) Based on IAT, the proposed watermarking scheme has implemented mutual conversion of coordinates from watermarked carrier and original watermark, and then it makes full use of the chaotic scrambling feature of AT to distribute the watermark into the carrier image. In the combination of the new space domain transformation and FSSVM, the watermarked carrier image still can remember the relationship among the local pixels after experiencing various attacks and realize the correct extraction of watermark. Therefore, the embedding and extraction methods have strong robustness to 
all kinds of conventional image attacks, and they also balance imperceptibility and robustness of watermark well.

\section{Conclusion}

In this paper, a detailed investigation of image watermarking process by handling it as an optimization procedure based on IAT and FSSVM is presented. The watermarking technique of spatial domain by using IAT is introduced to determine the positions embedded with watermark. Then, one can make full use of the scrambling feature of IAT to evenly distribute the watermark into the whole space of carrier image. Through increasing the secret key parameters, the security degree is improved efficiently. By using quantization and round methods in mathematics to change the pixels of embedding positions, it not only realizes the imperceptibility of watermark, but also deduces the quantitative extraction rules reversely. So the process realizes the blind extraction of watermark without depending on the original carrier image. Furthermore, FSSVM model is constructed to predict the original pixel values of watermarked carrier when the watermark is extracted. Through comparing the predictable values with the actual pixel values of the watermarked carrier image, the positions embedded with watermark can be found out easily. It makes full use of spatial domain features of the image and improves the accuracy and efficiency in predicting outcomes. What is more, the combination of spatial domain and SVM not only efficiently improves the robustness of watermark obviously, but also realizes the blind extraction of watermark. Thus, it achieves the efficacy of advantageous complementarities. Hence, the proposed scheme in this paper is different from the traditional watermark embedding and extraction methods. Theoretical analysis and computer simulations indicate the feasibility of our proposed algorithms. Therefore, our proposed scheme has satisfied the blind extraction, robustness, imperceptibility, and security requirements. In addition, to extend the proposed idea to color video watermarking is another future work.

\section{Conflict of Interests}

The authors declare that there is no conflict of interests regarding the publication of this paper.

\section{Acknowledgments}

This work was supported by the National Natural Science Foundation of China (nos. 61370169, 61402153, and 61472042), the Key Project of Science and Technology Department of Henan Province (no. 142102210056), the Science and Technology Research Key Project of Educational Department of Henan Province (nos. 13A520529, 12A520027), and the Education Fund for Youth Key Teachers of Henan Normal University.

\section{References}

[1] G. A. Papakostas, E. D. Tsougenis, and D. E. Koulouriotis, "Moment-based local image watermarking via genetic optimization," Applied Mathematics and Computation, vol. 227, pp. 222-236, 2014.

[2] B.-Z. Li and Y.-P. Shi, "Image watermarking in the linear canonical transform domain," Mathematical Problems in Engineering, vol. 2014, Article ID 645059, 9 pages, 2014.

[3] N. M. Makbol and B. E. Khoo, "Robust blind image watermarking scheme based on redundant discrete wavelet transform and singular value decomposition," AEU-International Journal of Electronics and Communications, vol. 67, no. 2, pp. 102-112, 2013.

[4] Y. Naderahmadian and S. Hosseini-Khayat, "Fast and robust watermarking in still images based on QR decomposition," Multimedia Tools and Applications, vol. 72, no. 3, pp. 2597-2618, 2014.

[5] M. Ali, C. W. Ahn, and M. Pant, "A robust image watermarking technique using SVD and differential evolution in DCT domain," Optik-International Journal for Light and Electron Optics, vol. 125, no. 1, pp. 428-434, 2014.

[6] A. Mishra, C. Agarwal, A. Sharma, and P. Bedi, "Optimized gray-scale image watermarkingusing DWT-SVD and firefly algorithm," Expert Systems with Applications, vol. 41, no. 17, pp. 7858-7867, 2014

[7] E. Nezhadarya and R. K. Ward, "Multiscale derivative transform and its application to image watermarking," Digital Signal Processing, vol. 33, pp. 148-155, 2014.

[8] M. Mundher, D. Muhamad, A. Rehman, T. Saba, and F. Kausar, "Digital watermarking for images security using discrete slantlet transform," Applied Mathematics \& Information Sciences, vol. 8, no. 6, pp. 2823-2830, 2014.

[9] A. K. Singh, M. Dave, and A. Mohan, "Hybrid technique for robust and imperceptible image watermarking in DWT-DCTSVD domain," National Academy Science Letters, vol. 37, no. 4, pp. 351-358, 2014.

[10] L. Sui and B. Gao, "Color image encryption based on gyrator transform and Arnold transform," Optics and Laser Technology, vol. 48, pp. 530-538, 2013.

[11] Z. Liu, L. Xu, T. Liu et al., "Color image encryption by using Arnold transform and color-blend operation in discrete cosine transform domains," Optics Communications, vol. 284, no. 1, pp. 123-128, 2011.

[12] M. R. Abuturab, "Securing color information using Arnold transform in gyrator transform domain," Optics and Lasers in Engineering, vol. 50, no. 5, pp. 772-779, 2012.

[13] L. F. Chen, D. M. Zhao, and F. Ge, "Image encryption based on singular value decomposition and Arnold transform in fractional domain," Optics Communications, vol. 291, pp. 98103, 2013.

[14] Y.-L. Chen, H.-T. Yau, and G.-J. Yang, "A maximum entropybased chaotic time-variant fragile: watermarking scheme for image tampering detection," Entropy, vol. 15, no. 8, pp. 31703185, 2013.

[15] S. Sadasivam, P. Moulin, and T. P. Coleman, "A message-passing approach to combating desynchronization attacks," IEEE Transactions on Information Forensics and Security, vol. 6, no. 3, pp. 894-905, 2011.

[16] X.-Y. Wang, E.-N. Miao, and H.-Y. Yang, "A new SVM-based image watermarking using Gaussian-Hermite moments," Applied Soft Computing, vol. 12, no. 2, pp. 887-903, 2012. 
[17] H.-Y. Yang, X.-Y. Wang, and C.-P. Wang, "A robust digital watermarking algorithm in undecimated discrete wavelet transform domain," Computers and Electrical Engineering, vol. 39, no. 3, pp. 893-906, 2013.

[18] L. D. Li, S. S. Li, A. Abraham, and J. S. Pan, "Geometrically invariant image watermarking using Polar Harmonic Transforms," Information Sciences, vol. 199, pp. 1-19, 2012.

[19] I. Nasir, F. Khelifi, J. Jiang, and S. Ipson, "Robust image watermarking via geometrically invariant feature points and image normalisation," IET Image Processing, vol. 6, no. 4, pp. 354-363, 2012.

[20] X. Wang, Z. Xu, and P. Niu, "A feature-based digital watermarking scheme for halftone image," AEU: International Journal of Electronics and Communications, vol. 64, no. 10, pp. 924-933, 2010.

[21] Y. Fu, R. Shen, and H. Lu, "Watermarking scheme based on support vector machine for colour images," Electronics Letters, vol. 40, no. 16, pp. 986-987, 2004.

[22] H.-H. Tsai and D.-W. Sun, "Color image watermark extraction based on support vector machines," Information Sciences, vol. 177, no. 2, pp. 550-569, 2007.

[23] C. H. Li, Z. D. Lu, and K. Zhou, "An image watermarking technique based on support vector regression," in Proceedings of the International Symposium on Communications and Information Technologies (ISCIT '05), pp. 177-180, October 2005.

[24] H. Peng, J. Wang, and W. X. Wang, "Image watermarking method in multiwavelet domain based on support vector machines," Journal of Systems and Software, vol. 83, no. 8, pp. 1470-1477, 2010.

[25] H.-H. Tsai, H.-C. Tseng, and Y.-S. Lai, "Robust lossless image watermarking based on $\alpha$-trimmed mean algorithm and support vector machine," Journal of Systems and Software, vol. 83, no. 6, pp. 1015-1028, 2010.

[26] C.-H. Li, H.-F. Ling, and Z.-D. Lu, "Semi-fragile watermarking based on SVM for image authentication," in IEEE International Conference onMultimedia and Expo (ICME '07), pp. 1255-1258, July 2007. 


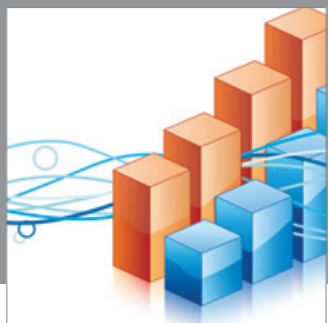

Advances in

Operations Research

mansans

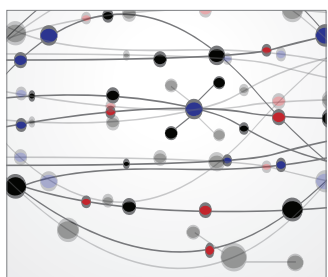

The Scientific World Journal
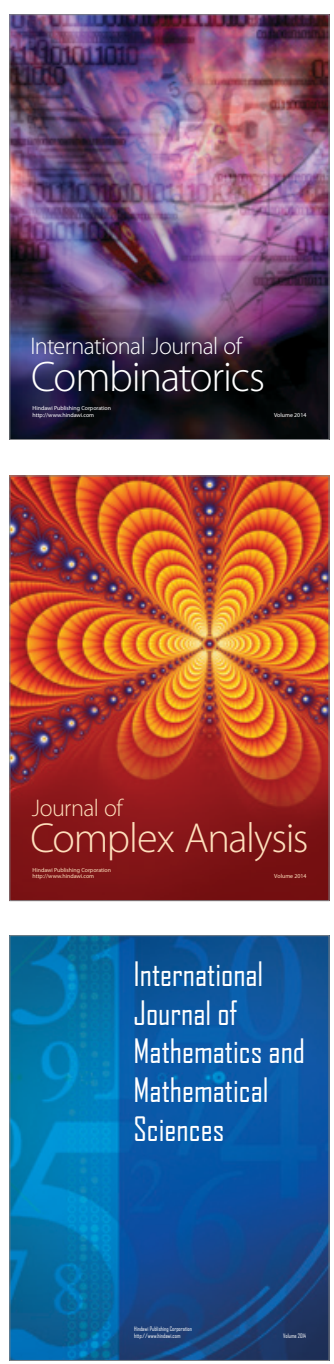
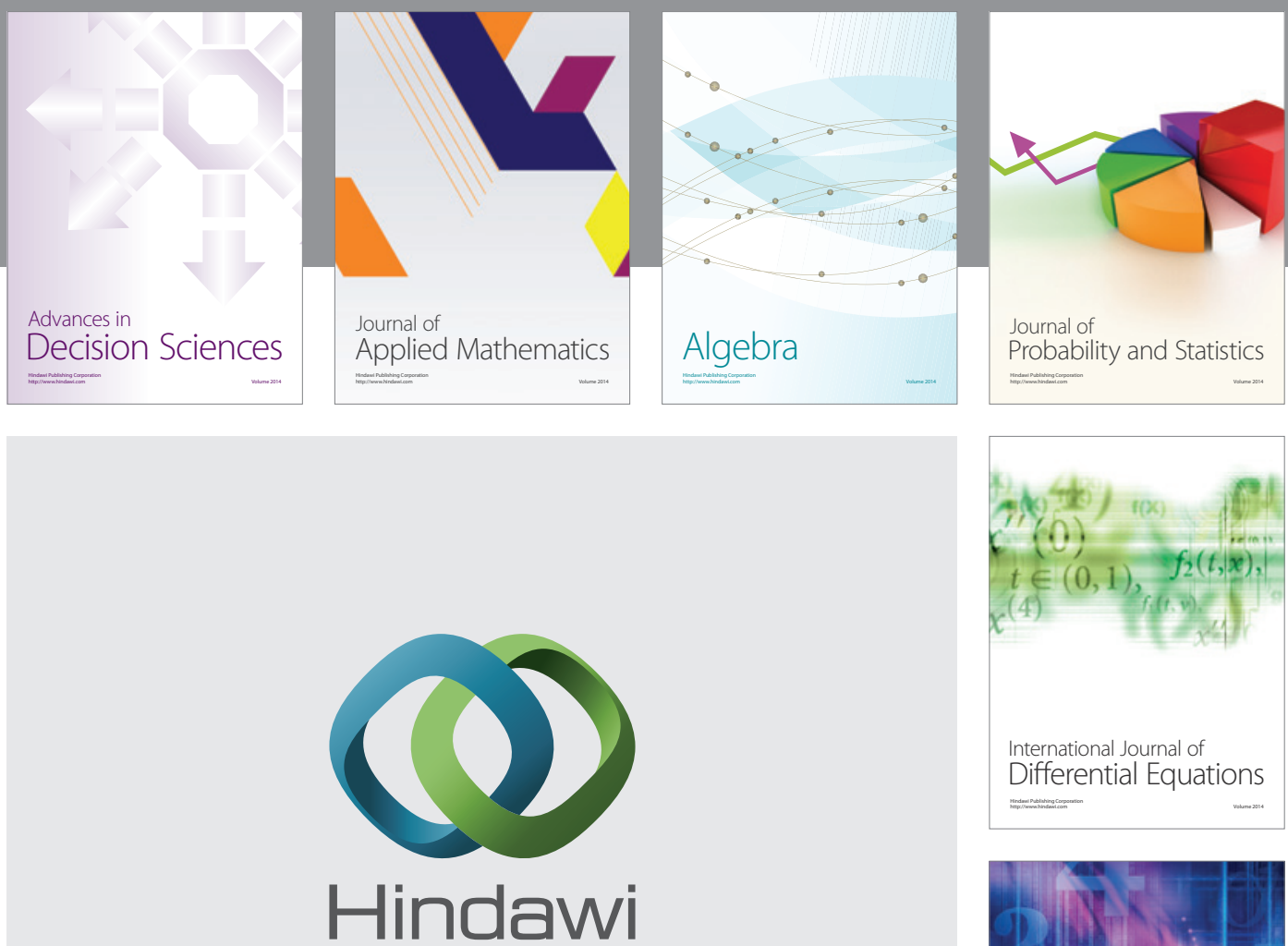

Submit your manuscripts at http://www.hindawi.com
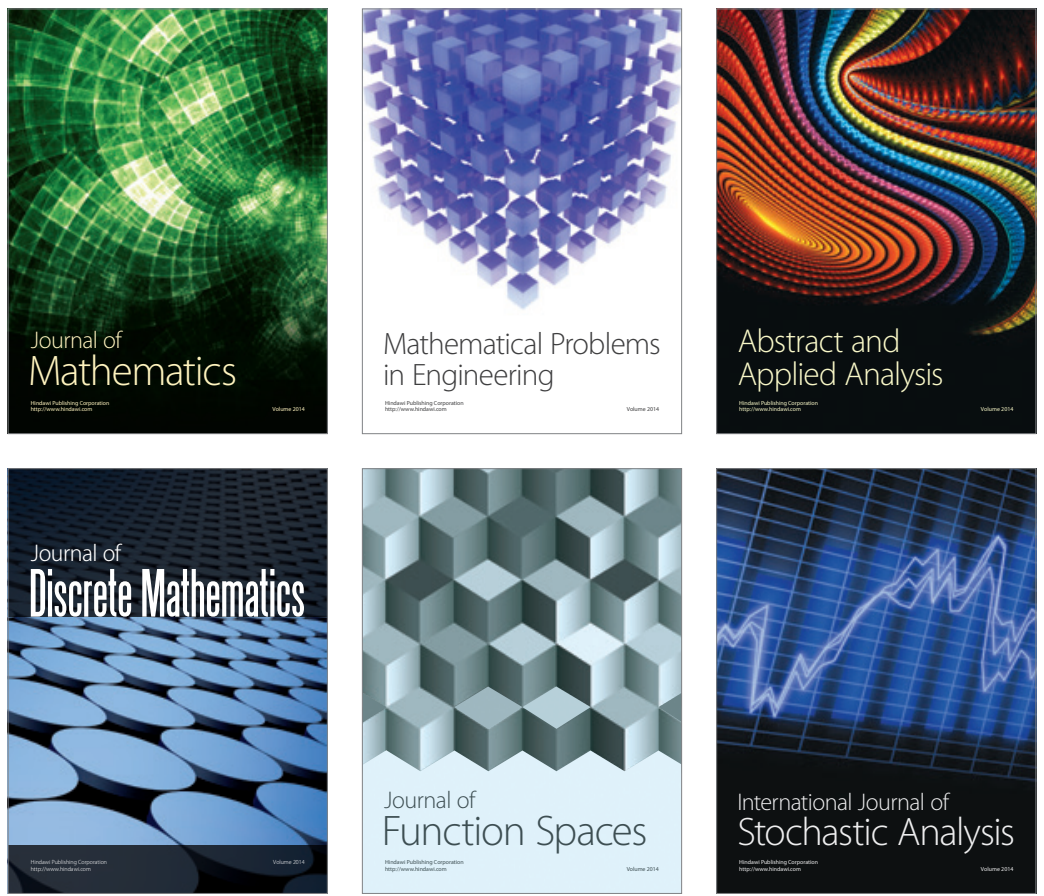

Journal of

Function Spaces

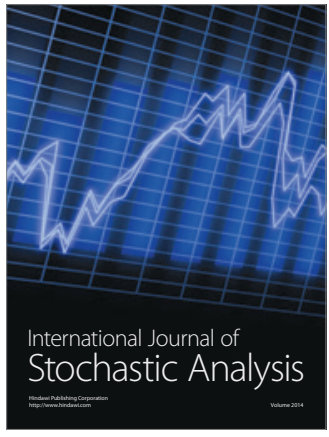

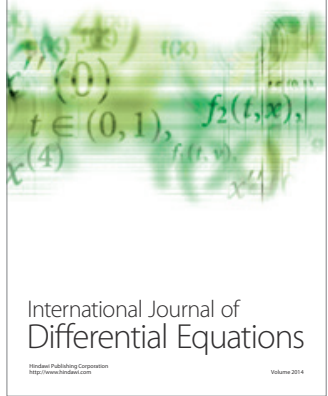
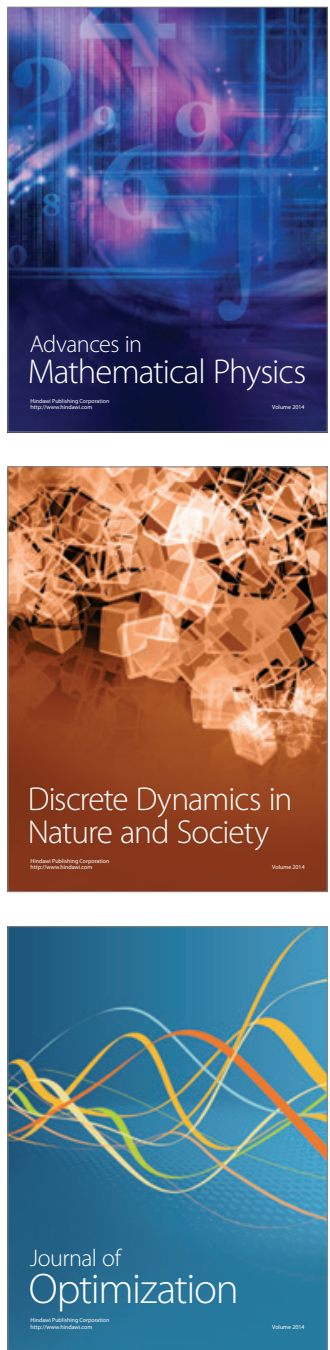